\title{
Universality of electron distributions in high-energy air showers - description of Cherenkov light production
}

\author{
F. Nerling ${ }^{a, \dagger}$, J. Blümer $^{a, b}$, R. Engel ${ }^{a}$, M. Risse ${ }^{a}$ \\ ${ }^{a}$ Forschungszentrum Karlsruhe, Institut für Kernphysik \\ ${ }^{b}$ Universität Karlsruhe, Institut für Experimentelle Kernphysik \\ ${ }^{\dagger}$ Corresponding author, Email: frank.nerling@ik.fzk.de
}

\begin{abstract}
The shower simulation code CORSIKA has been used to investigate the electron energy and angular distributions in high-energy showers. Based on the universality of both distributions, we develop an analytical description of Cherenkov light emission in extensive air showers, which provides the total number and angular distribution of photons. The parameterisation can be used e.g. to calculate the contribution of direct and scattered Cherenkov light to shower profiles measured with the air fluorescence technique.
\end{abstract}

Key words: ultrahigh-energy cosmic rays, extensive air showers, Cherenkov light, air fluorescence technique, electron energy spectra, electron angular distributions PACS: $96.40 . \mathrm{Pq}, 41.60 . \mathrm{Bq}, 13.85 . \mathrm{Tp}$

\section{Introduction}

The measurement of ultrahigh-energy air showers above $10^{17} \mathrm{eV}$ is one of the main tools to understand the nature, origin and propagation of cosmic rays at highest energies. Large-scale experiments, like the Pierre Auger Observatory [1], AGASA [2], HiRes [3], and Telescope Array [4] focus on the precise determination of the energy spectrum, mass composition and arrival direction distribution of ultrahigh-energy cosmic rays (UHECR).

The calorimetric measurement of the longitudinal shower profile with the fluorescence technique is one of the most direct methods to determine the primary energy [5] and also can be used to infer the primary particle type. The charged shower particles induce nitrogen fluorescence light, which is emitted isotropically mainly in the near UV-range of 300-400 nm [6]. Assuming the 
fluorescence yield is proportional to the energy deposit by a shower, as indicated by measurements $[7,8]$, observing the emitted fluorescence light gives a measure of the local ionisation energy deposit and hence the shower size.

However, charged particles in extensive air showers (EAS) are mostly relativistic and Cherenkov light is produced as well. In contrast to the fluorescence light, Cherenkov photons are emitted mostly in the forward direction. Depending on the observation angle with respect to the shower axis, the intensity of produced Cherenkov light contributes a non-negligible signal in fluorescence detectors $[5,9,10,11]$, which can be of same order as the fluorescence light signal itself $[10,11,12]$. Thus, an efficient correction for or an explicit consideration of the Cherenkov contribution to the measured signal is needed for the determination of the primary particle properties.

In the pioneering Fly's Eye experiment, Cherenkov light was estimated [5] based on Hillas' simulations of $100 \mathrm{GeV}$ photon showers [13]. Assuming universality of electron distributions in EAS, the parameterisation in shower age developed by Hillas was applied to showers of ultrahigh-energy [5,14,15]. More recent simulation studies at higher energies and with hadron primaries confirm such an universality of electron energy spectra $[16,18]$.

During the last decades substantial progress has been made in the simulation of very high energy EAS. New simulation packages such as CORSIKA [19] and modern high-energy interaction models allow a more reliable prediction of particle distributions in showers. At the same time the precision of UHECR detectors has increased dramatically, requiring a treatment of Cherenkov light production as accurate as possible.

In this work we perform a systematic study of shower particle distributions relevant to Cherenkov light calculation. A complete model for Cherenkov light calculation is developed and compared to predictions from CORSIKA employing QGSJET 01 [20]. In this approach, the total Cherenkov light can be calculated as function either of the shower size profile or the energy deposit profile. Parameterisations of electron energy spectra and Cherenkov photon angular distributions in high-energy showers are developed, which serve as a basis for calculations of radiation emitted by air showers and may also be useful for other purposes. A direct application is the prediction of Cherenkov radiation as needed for reconstruction of longitudinal shower size profiles measured by experiments based on the fluorescence technique.

The outline of the paper is as follows. In Sec. 2, an analytical expression is derived for calculating Cherenkov light production in EAS based on electrons ${ }^{1}$ only. In Sec.3, electron energy spectra are shown to be universal, parameterised in terms of a phenomenological definition of the shower age parameter

$\overline{1}$ Throughout this work, the notation 'electrons' include $e^{+}$and $e^{-}$. 
and applied for calculating the longitudinal Cherenkov light profile; the results are compared to other approaches and to a detailed CORSIKA simulation. Angular distributions of electrons and Cherenkov photons are investigated in Sec. 4. The former are shown to be universal and the latter are parameterised in dependence of altitude and shower age. Finally, the concept of ionisation energy deposit in simulation and reconstruction of fluorescence light profiles is discussed in Sec. 5. Part of the results presented here have already been shown in $[12,18,21]$.

\section{Calculation of Cherenkov light production}

The total number of Cherenkov photons $\mathrm{d} N_{\gamma}$ produced in a shower per interval of slant depth $\mathrm{d} X$ and angle $\mathrm{d} \theta$ with respect to the shower axis is given by

$$
\frac{\mathrm{d} N_{\gamma}}{\mathrm{d} X \mathrm{~d} \theta}(X, \theta, h)=\sum_{i=\mathrm{e}, \mu, \ldots} A_{\gamma}^{i}(X, \theta, h) \int_{E_{\mathrm{thr}}^{i}}^{\infty} \frac{\mathrm{d} N_{i}}{\mathrm{~d} E}(X) y_{\gamma}^{i}(h, E) \mathrm{d} E
$$

where $\mathrm{d} N_{i} / \mathrm{d} E(X)$ are the normalised differential energy spectra of corresponding charged particles of type $i$ (= electrons, muons, ...) at depth $X$, $E_{\mathrm{thr}}^{i}(h)$ the height-dependent Cherenkov energy thresholds, and $y_{\gamma}^{i}(h, E)$ the corresponding Cherenkov photon yields. $A_{\gamma}^{i}(X, \theta, h)$ are the normalised angular distributions of produced Cherenkov photons with respect to the shower axis. The photons are assumed to be produced at the shower axis, which is a good approximation for fluorescence technique applications since most charged particles are moving at a lateral distance of less than $60 \mathrm{~m}$.

The amount of so-called scattered Cherenkov light reaching a detector depends mostly on the total number of photons produced since the dominant Rayleigh scattering process is nearly isotropic. Therefore, the integral $\frac{\mathrm{d} N_{\gamma}}{\mathrm{d} X}(X, h)$, corresponding to integration of Eq. (1) over all angles $\theta$, is the most important quantity and typically used to calculate the scattered Cherenkov light contribution in fluorescence measurements. The so-called direct Cherenkov light contribution, i.e. Cherenkov photons hitting directly the detector without scattering in the atmosphere, is determined by both the total Cherenkov light intensity and the angular distributions $A_{\gamma}^{i}(X, \theta, h)$.

The number of Cherenkov photons produced by a charged particle of total energy $E$ and charge $Z$ in a wavelength interval between $\lambda_{1}$ and $\lambda_{2}$ is given by

$$
y_{\gamma}:=\frac{\mathrm{d} N_{\gamma}^{(1)}}{\mathrm{d} X}(h, E)=\frac{2 \pi \alpha Z^{2}}{\rho(h)} \int_{\lambda_{1}}^{\lambda_{2}}\left(1-\frac{1}{n^{2}(h, \lambda) \beta^{2}}\right) \frac{\mathrm{d} \lambda}{\lambda^{2}}
$$


where $\alpha$ is the fine-structure constant, $\beta=v / c, \rho$ the air density at height $h$, and $n$ the refractive index. Measuring $\mathrm{d} X$ along the shower trajectory we introduce the approximation that all particle trajectories are parallel to the shower axis, resulting in an under-estimation of produced Cherenkov photons of order one percent, cf. Sec.3.4. The wavelength interval is usually about 300-400 nm for fluorescence telescopes [1,5]. Since in air $n \approx 1$ and dispersion is negligible the integrand of (2) can be approximated as

$$
1-(\beta n)^{-2}=1-\left(1-\frac{m^{2} c^{4}}{E^{2}}\right)^{-1}(1+\delta)^{-2} \approx 2 \delta-\frac{m^{2} c^{4}}{E^{2}},
$$

where $\delta(h)=n(h)-1$, and $m$ the charged particle mass. The energy threshold condition for Cherenkov radiation in air reads

$$
E_{\mathrm{thr}}(h)=m \mathrm{c}^{2} / \sqrt{2 \delta(h)}
$$

giving $21 \mathrm{MeV}$ for electrons and $4.4 \mathrm{GeV}$ for muons at sea level. These thresholds increase to $37 \mathrm{MeV}$ and $7.6 \mathrm{GeV}$ respectively at an altitude of $10 \mathrm{~km}$ (US standard atmosphere).

Particles heavier than electrons contribute less than $2 \%$ to the shower size around the shower maximum even for heavy primaries and their energy threshold for Cherenkov light production is very high. Therefore, to a good approximation, practically all charged particles can assumed to be electrons in the following considerations. Expression (1) simplifies to the following ansatz

$$
\frac{\mathrm{d} N_{\gamma}}{\mathrm{d} X \mathrm{~d} \theta}(X, \theta, h)=A_{\gamma}(X, \theta, h) \cdot N(X) \int_{\ln E_{\mathrm{thr}}} y_{\gamma}(h, E) f_{\mathrm{e}}(X, E) \mathrm{d} \ln E
$$

Here, $N(X)$ is the charged particle number as function of depth $X$, and $E_{\text {thr }}$ the local Cherenkov energy threshold for electrons. For a given shower geometry, $h=h(X)$ follows from the atmospheric model assumed, $f_{\mathrm{e}}(X, E)$ is the normalised differential electron energy spectrum at depth $X$

$$
f_{\mathrm{e}}(X, E)=\frac{1}{N_{\mathrm{e}}(X)} \frac{\mathrm{d} N_{\mathrm{e}}}{\mathrm{d} \ln E}(X, E)
$$

and $A_{\gamma}(X, \theta, h)$ is the normalised angular distribution of all Cherenkov photons produced.

We show in Sec. 3 and 5 that the ansatz (5) describes well the Monte Carlo results used as reference. 


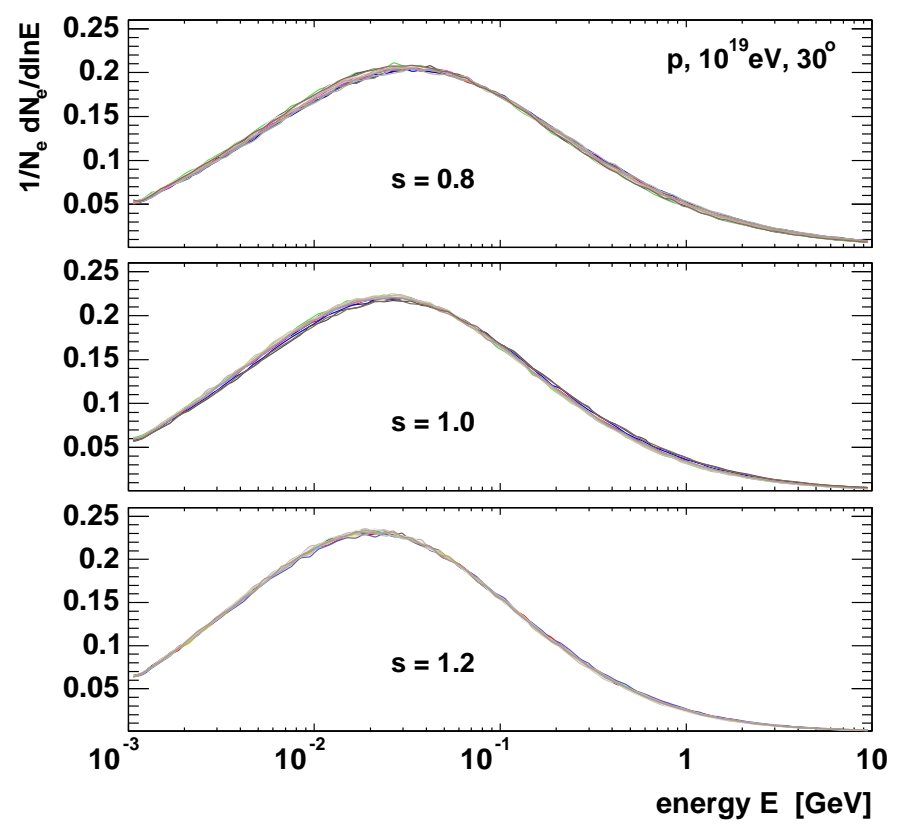

Fig. 1. Electron energy spectra obtained with CORSIKA for 3 different fixed shower ages. Shown are 15 individual proton showers of $10^{19} \mathrm{eV}$.

\subsection{Monte Carlo simulations}

The cosmic ray simulation code CORSIKA [19] version 6.137 with the hadronic interaction models QGSJET 01 [20] for high-energy interactions and GHEISHA $2002[22,23]$ for low-energy interactions has been used to study the development of high-energy showers. Energy spectra of electrons and angular distributions of electrons and Cherenkov photons were obtained for individual showers and different combinations of primary energy, zenith angle and primary particle type. The US standard atmosphere [24,25] has been used as atmospheric model. Also the longitudinal shower size profile and produced Cherenkov photon profile, which are needed to test the analytical model proposed in this paper, are calculated in the simulations. In CORSIKA calculations, the wavelength dependence of the refractive index $n(\lambda)$ is neglected. This simplification is a good approximation as has been shown by a comparison [11] to GEANT 3.21 [26] simulations taking into account the wavelength dependence. To reduce CPU-time, optimum thinning [27,28] of $10^{-6}$ has been applied unless otherwise noted. The UPWARD option has been enabled to follow the upward going electromagnetic particles ${ }^{2}$. Low-energy thresholds of $100 \mathrm{MeV}$ for hadrons and muons, and of $1 \mathrm{MeV}$ for electrons and gamma-rays

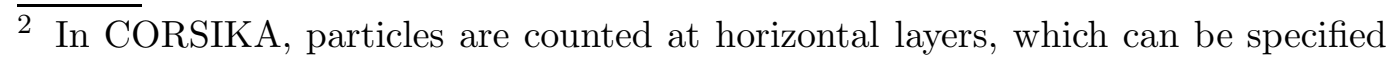
by the user. By default, particles with tracks at angles larger than $90^{\circ}$ to the vertical are not followed. By applying the UPWARD option, these particles are treated in the electromagnetic shower component. 


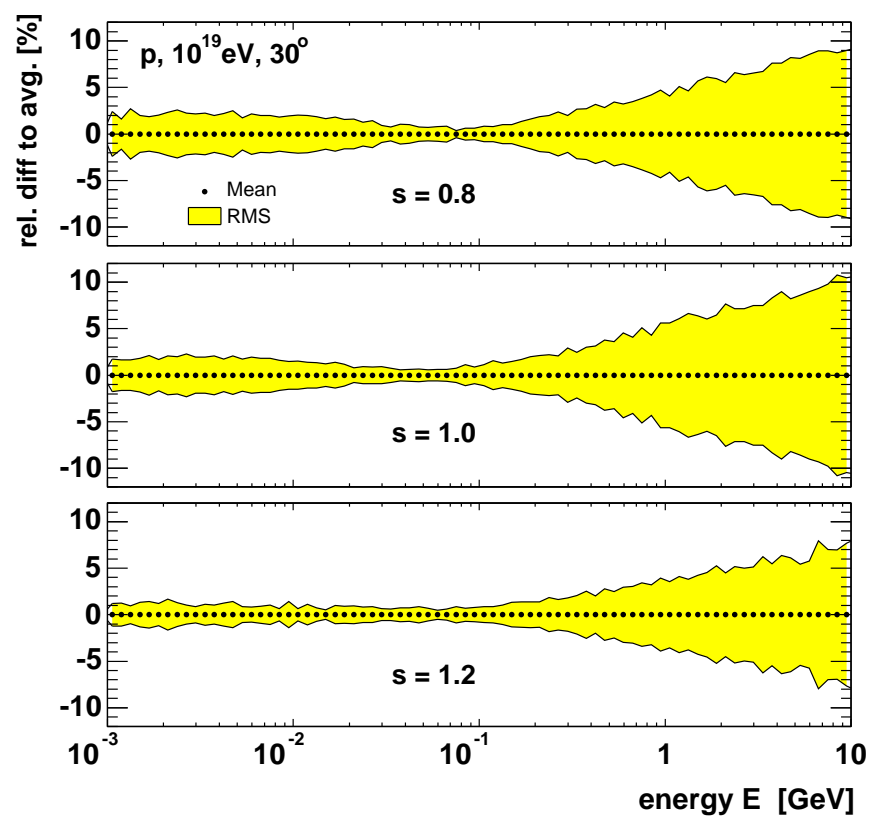

Fig. 2. Shower-to-shower fluctuations of electron energy spectra. The shaded bands indicate the RMS characterising the deviations of the individual showers of Fig. 1 from the average.

have been used, unless otherwise noted.

\section{Electron energy spectra}

In Figs. 1 and 2, normalised differential electron energy spectra $f_{\mathrm{e}}(X, E)$ of individual showers obtained with CORSIKA are compared for fixed (effective) shower age

$$
s=\frac{3}{\left(1+2 X_{\max } / X\right)},
$$

where $X$ denotes the slant depth (in $\mathrm{g} / \mathrm{cm}^{2}$ ). The study covers the shower age range $0.8 \leq s \leq 1.2$ that is most important for fluorescence observations. The spectra have been normalised according to

$$
f_{\mathrm{e}}(X, E)=\frac{1}{N_{\mathrm{e}}} \frac{\mathrm{d} N_{\mathrm{e}}}{\mathrm{d} \ln E}, \quad \text { with } \int_{\ln E_{\text {cut }}} f_{\mathrm{e}}(X, E) \mathrm{d} \ln E=1,
$$

where $E_{\text {cut }}$ is the energy threshold adopted in the simulation $(1 \mathrm{MeV}$ in the examples shown). The importance of the low-energy threshold $E_{\text {cut }}$ for normalisation and Cherenkov (and fluorescence) light calculations is discussed in more detail below. If the longitudinal development is described in terms of 


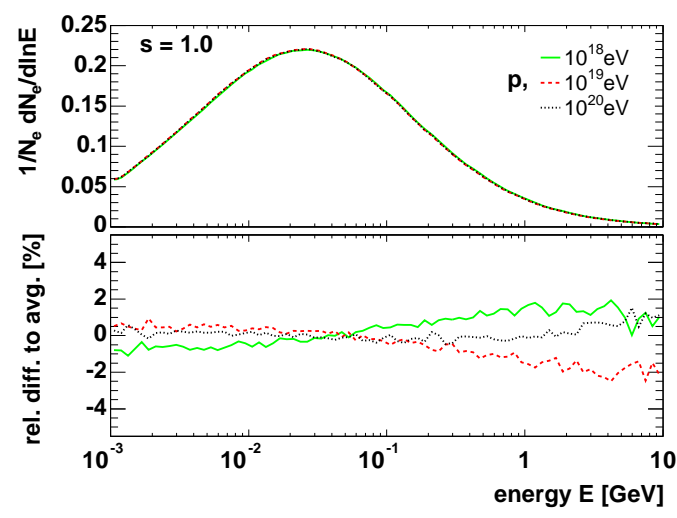

Fig. 3. Universality of electron energy spectra - different primary energy. In shower age, mean showers of $10^{18}, 10^{19}$ and $10^{20} \mathrm{eV}$ (each curve represents the mean distributions of 15 individual showers) do not show significant differences in their electron energy spectra; here shown for $s=1.0$ and proton initiated showers.

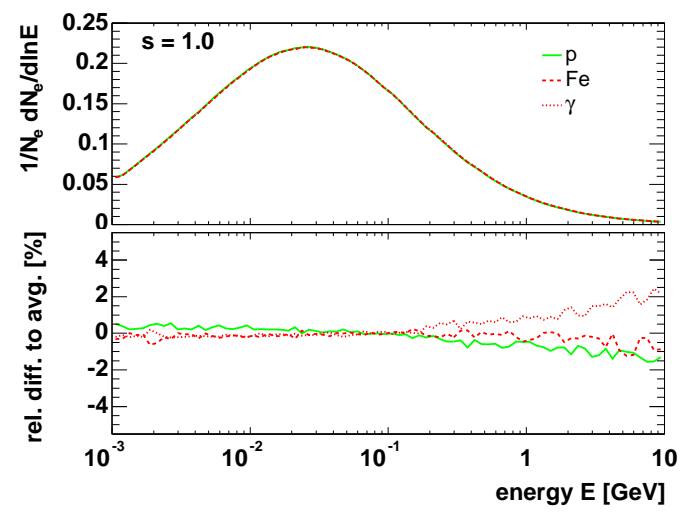

Fig. 4. Universality of electron energy spectra - different primary particle type. In shower age, mean proton, iron and gamma-ray showers of energies $>10^{17} \mathrm{eV}$ (each curve represents the mean distribution averaged over $10^{18}, 10^{19}$, and $10^{20} \mathrm{eV}$ ) do not show significant differences in their electron energy spectra, here shown for $s=1.0$.

shower age, the remaining shower-to-shower fluctuations are relatively small and due mainly to the depth of the first interactions. Deviations are smallest beyond the shower maximum because the individual distributions are the more insensitive to initial differences in shower development the older the shower is. The small fluctuations of the envelopes in Fig. 2 are of statistical kind and caused by the thinning applied in the simulations. The comparison is shown here only for proton showers of $10^{19} \mathrm{eV}$ for which the fluctuations are the largest and looks similar for different primary particle types at ultrahighenergy $\left(>10^{17} \mathrm{eV}\right)$.

\subsection{Universality}

Average electron energy spectra $f_{\mathrm{e}}(E, s)$ for proton showers of different primary energies and showers of different primary particle types (proton, iron, and gamma-ray averaged over different energies $10^{18}, 10^{19}$, and $10^{20} \mathrm{eV}$ ) are shown ${ }^{3}$ in Figs. 3 and 4. For the large electron energy range from $1 \mathrm{MeV}$ up to $10 \mathrm{GeV}$, the mean distributions are similar when normalised according to (8). The energy distribution of electrons in UHECR initiated EAS does not depend significantly either on primary energy or on primary particle type, which allows a universal parameterisation in shower age $[16,18]$. It is also indepen-

$\overline{3}$ In the case of gamma-ray showers, simulations were done for $10^{19.5} \mathrm{eV}$ instead of $10^{20} \mathrm{eV}$ as otherwise primary gamma-rays interact with the Earth magnetic field well before reaching the atmosphere (pre-shower effect) [29]. 


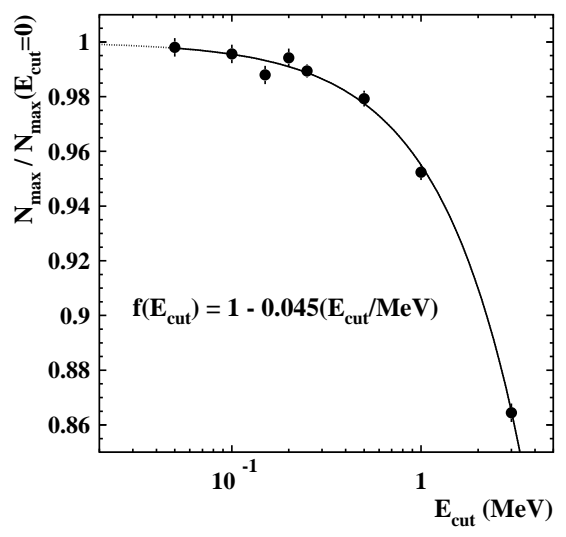

Fig. 5. Dependence of shower size at maximum on the simulation energy threshold. The ratio of the mean shower size at shower maximum $N_{\max }$ to that extrapolated to $E_{\text {cut }}=0$ is shown for different simulation thresholds. A parameterisation of this ratio as a function of $E_{\text {cut }}$ is given [18].

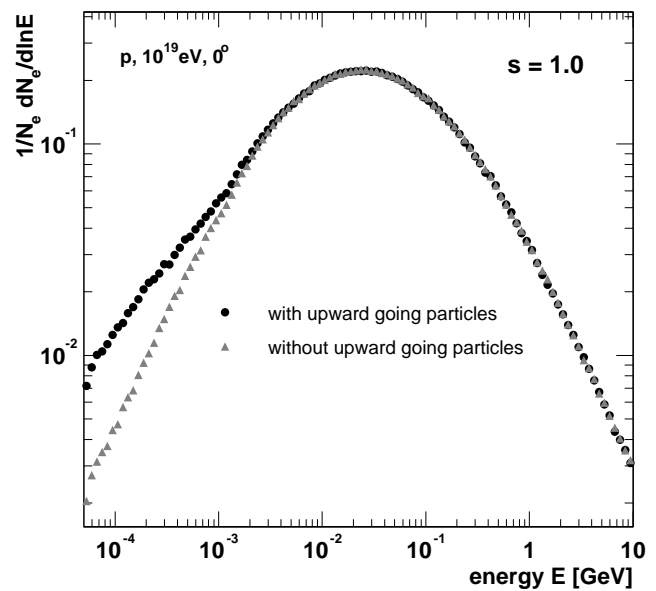

Fig. 6. Electron energy spectra obtained from CORSIKA $\left(E_{\text {cut }}=50 \mathrm{keV}\right)$ with and without upward going particles. The spectra are normalised according to Eq. (8) assuming $E_{\text {cut }}=1 \mathrm{MeV}$, for discussion see text.

dent of the shower zenith angle, however, this has only been investigated for inclinations smaller up to $60^{\circ}$. Increasing deviations from the spectral shape shown occur at $60^{\circ}$ for high-energy electrons in the GeV-range [12].

\subsection{Problem of low-energy cut and definition of shower size in simulations}

In general, simulating air showers is not possible without applying a low-energy cut $E_{\text {cut }}$ on the tracked particles as the number of produced photons diverges for $E_{\text {cut }} \rightarrow 0$ [30]. The values of $E_{\text {cut }}$ that are typically applied in simulations are in the range of $100 \mathrm{keV}-3 \mathrm{MeV}$. Therefore, a parameterisation of electron energy spectra for the purpose of calculating Cherenkov light from simulated shower size profiles has to account for different energy cuts to be consistent with the simulation. Particularly in calculations of Cherenkov light based on ansatz (5), and for deriving energy deposit profiles to compute fluorescence light production, the low-energy cut applied in simulations is important since the number of charged particles provided by the simulations refers only to the particles above the threshold $E_{\text {cut }}$. The effect of different values of the simulation threshold on the shower size is illustrated in Fig. 5. For a detailed discussion of energy deposit calculations, see [31,32].

According to the definition of shower size, particles are counted when crossing virtual planes (horizontal in the CORSIKA version used). Depending on the angular distribution, particles might not be counted when their tracks are 


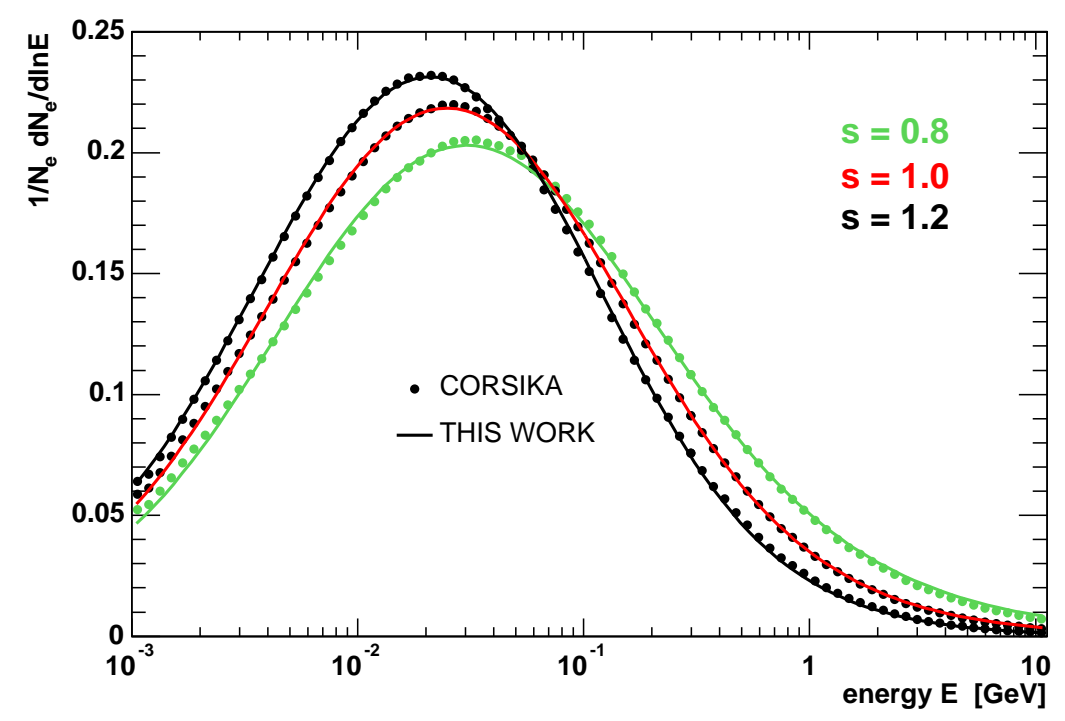

Fig. 7. Comparison of the new parameterisation Eq. (9), see also [18], and the energy spectra of an individual shower obtained with CORSIKA, proton, $10^{19} \mathrm{eV}$.

parallel to these planes and when they are going upwards. Multiple scattering of particles might cause low-energy particles (back-scattered) being counted multiply, which implies some ambiguity in the definition of shower size. This ambiguity is mostly related to low-energy particles as can be seen in Fig. 6 . The simulated electron energy spectrum of a vertical shower is shown for both upward going particles having been accounted for and not. Differences in the corresponding distributions occur for electrons below about $1 \mathrm{MeV}$ and increase with decreasing energy. Only electrons of less than about $1 \mathrm{MeV}$ are affected because of their angular distribution (with respect to the shower axis), which is broad in the low-energy range up to a few $\mathrm{MeV}$ and steepens with increasing energy, see discussion in Sec.4.1.

\subsection{Parameterisation in shower age}

Motivated by the high-energy limit of the energy behaviour of electrons in the cascade theory under approximation A [30] the following parameterisation

$$
f_{\mathrm{e}}(E, s)=a_{0} \cdot \frac{E}{\left(E+a_{1}\right)\left(E+a_{2}\right)^{s}},
$$

is proposed. As shown in Fig. 7, the CORSIKA spectra can be reproduced well by ansatz (9) using the parameters given in the appendix. The parameter $a_{0}$ follows automatically from the normalisation condition (8). The $E_{\text {cut }}{ }^{-}$ dependence of the normalisation $a_{0}$ is not negligible. In the energy range well 


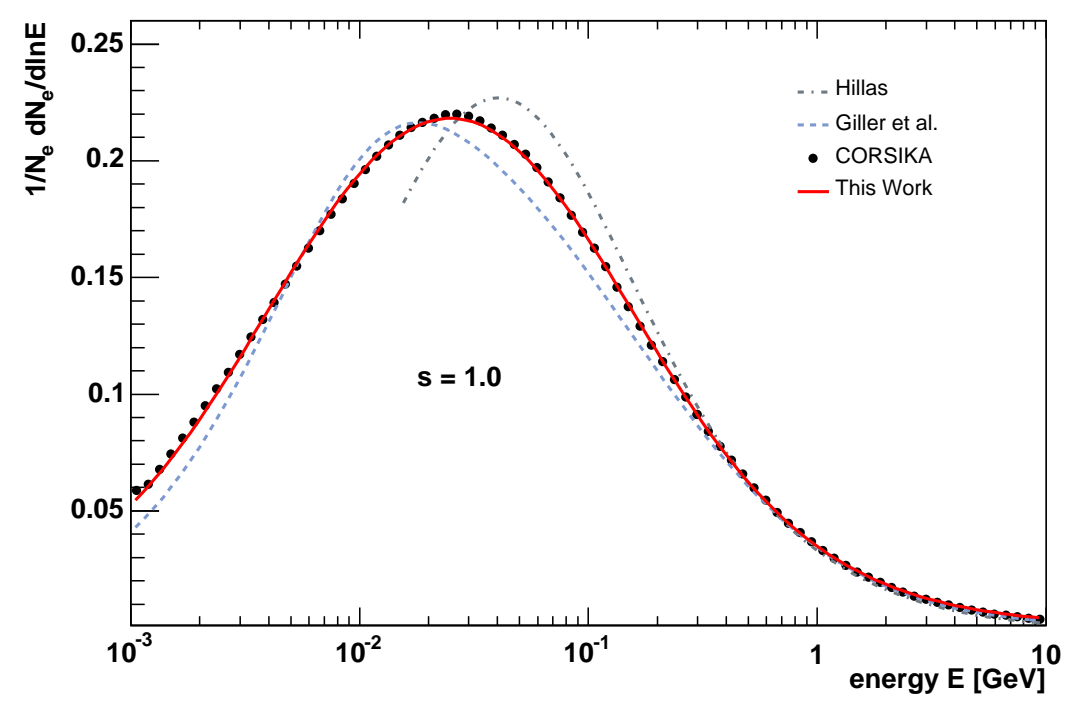

Fig. 8. Mean electron energy spectrum derived with CORSIKA (circles) for $s=1.0$. The Monte Carlo predictions are compared to the parameterisations according to Hillas [13] (dashed-dotted), the new parameterisation (Eq. 9) (solid) and Giller et al. [16] (dashed).

below $250 \mathrm{keV}$, this dependence might be negligible, but for larger cut-off values, the normalisation changes by up to e.g. about $10 \%$ in the energy cut range from $50 \mathrm{keV}$ to $2 \mathrm{MeV}$, see Fig. 5. A numerical expression for $a_{0}$ as a function of shower age $s$ and $E_{\text {cut }}$ is given in the appendix.

The parameterisation (9) is compared with other parameterisations and electron energy spectra obtained with CORSIKA in the following. In the case of Hillas' parameterisation, the normalisation integral cannot be calculated directly. Given the fact that this parameterisation was obtained for $100 \mathrm{GeV}$ primary photons, a larger disagreement to CORSIKA above $15 \mathrm{MeV}$ (the lower validity limit given in [13]) might have been expected. Another functional form is proposed by Giller et al. in [16]. Neither Hillas' nor the parameterisation given by Giller et al. take into account different low-energy thresholds. While in [13] $E_{\text {cut }}=50 \mathrm{keV}$ is given, no energy threshold has been specified in [16]. In the following, the parameterisations of Giller et al. is normalised the same way as the parameterisation introduced here, see Eq. (8), using $E_{\text {cut }}=1 \mathrm{MeV}$. In the case of the integral distribution provided by Hillas, the parameterisation is renormalised to compare properly. In this case the renormalisation is done according to the CORSIKA results on the dependence of shower particle content on simulation energy threshold, as displayed in Fig. 5.

In Fig. 8 and 9, all three approaches are compared to the CORSIKA predictions. The comparison is shown for a mean shower averaged in shower age over 87 individual showers of different primary energies and particle types $\left(10^{18}, 10^{19}\right.$, and $10^{20} \mathrm{eV} ; 45$ proton, 12 iron, and 30 gamma-ray induced show- 


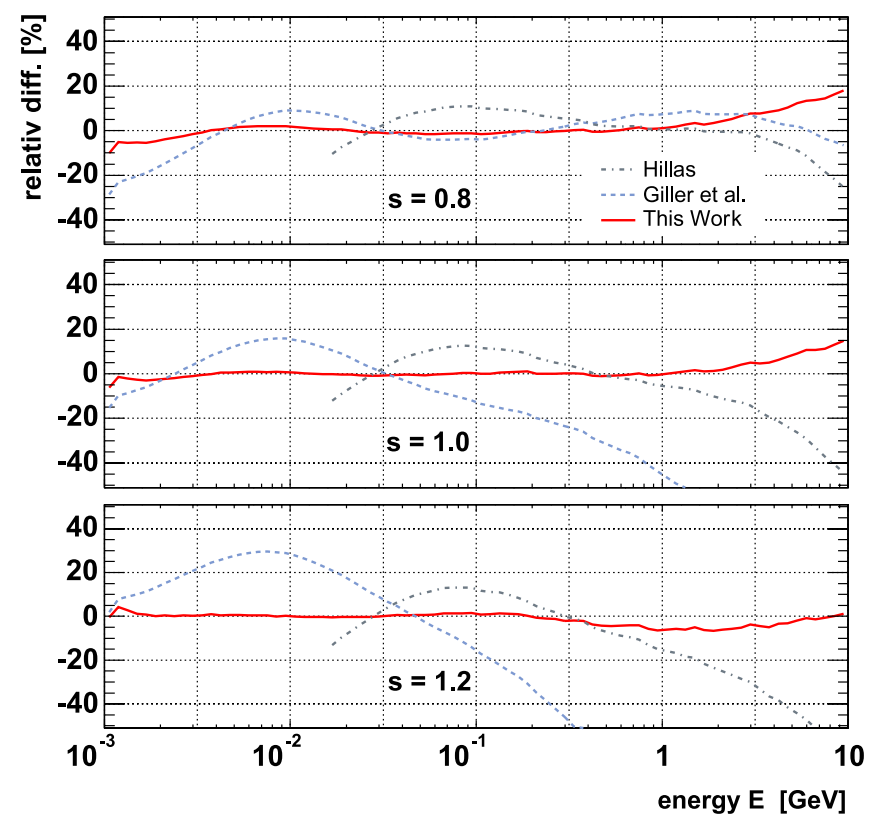

Fig. 9. Relative differences between model predictions and Monte Carlo results (relative to CORSIKA) as compared in Fig. 8 ([13] (dashed dotted), [16] (dashed), and the parameterisation (Eq. 9) (solid)).

ers, cf. Fig. 3 and Fig.4) at shower maximum. The other analytically calculated distributions show a remarkable shift of the maxima with respect to the CORSIKA result of about $25 \mathrm{MeV}$ at shower maximum (Hillas: $\sim 40 \mathrm{MeV}$, Giller et al.: $\sim 20 \mathrm{MeV}$ ), whereas the proposed parameterisation gives a very good overall description of the Monte Carlo results. The relative differences of the different approaches with respect to CORSIKA are shown for three stages of shower development in Fig. 9. The parameterisation (9) predicts the CORSIKA results within a few percent for the whole $\mathrm{MeV}$-range, whereas uncertainties increase up to about $20 \%$ at $10 \mathrm{GeV}$. In particular the energy range most important for Cherenkov light calculations $(20 \mathrm{MeV}$ to $1 \mathrm{GeV})$ is described with high accuracy.

\subsection{Total number of Cherenkov photons produced}

According to ansatz (5) the total number of produced Cherenkov photons can be calculated applying the parameterisation (9) of the electron energy spectrum $f_{\mathrm{e}}(E, s)$ and the charged particle number $N_{\mathrm{ch}}(X)$ provided by CORSIKA. In Fig. 10, the result is compared to the CORSIKA simulated profile and to analytical calculations based on the other analytical formulae for electron spectra [13,16] already considered in the previous section. Using the analytical expression going back to Hillas leads to an over-estimation of the Monte Carlo result, whereas the work of giller et al. [16] results in an under-estimation. The calculation labelled 'Hillas (s fixed)' employs the parameterisation of [13] for a 


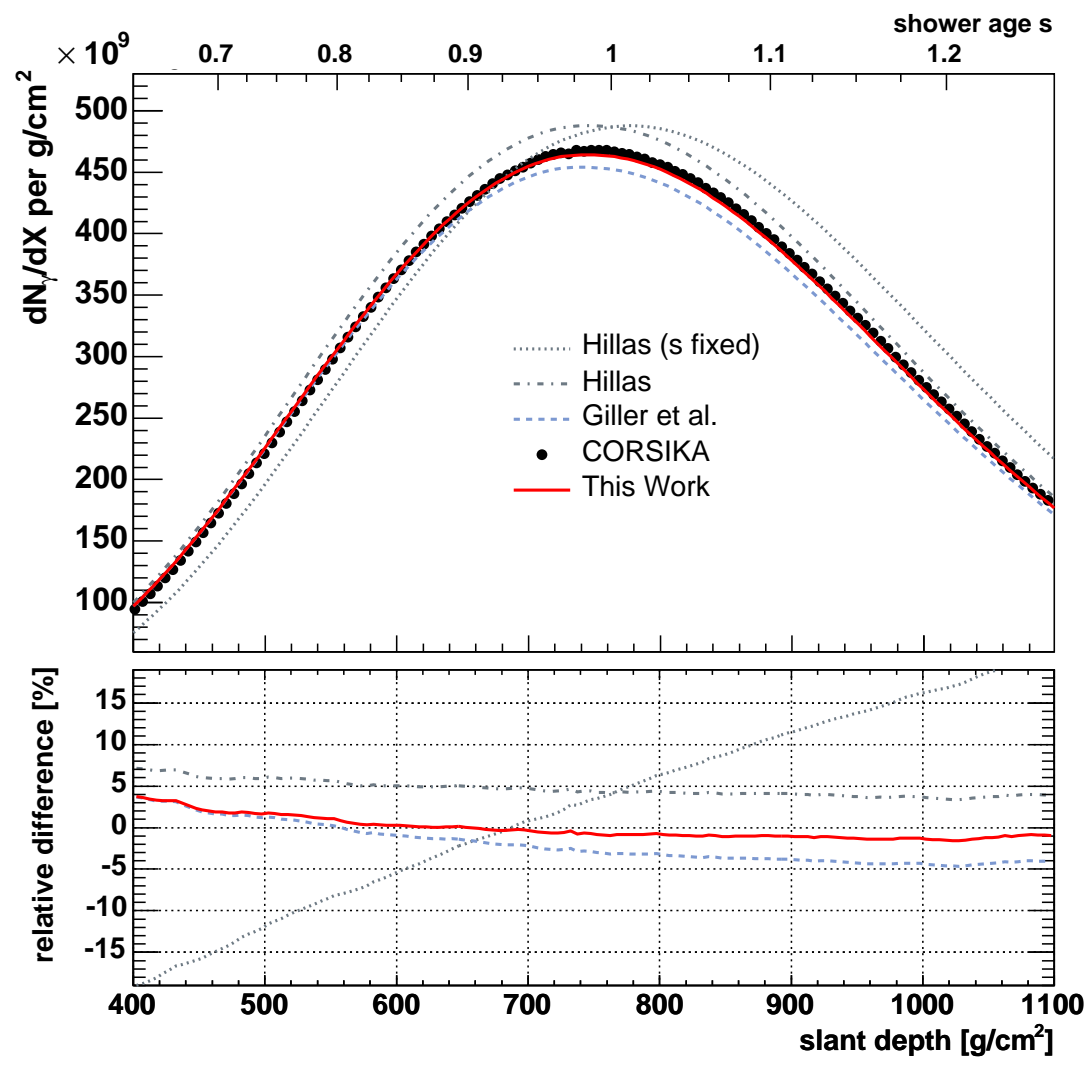

Fig. 10. Comparison of the total number of Cherenkov photons produced per slant depth within an individual shower (proton, $10^{19} \mathrm{eV}, 30^{\circ}$ ) simulated with CORSIKA and analytically calculated by ansatz (5) using different parameterisations of electron energy spectra. The different approaches are marked by dashed-dotted line for [13] and dotted line for the simplification $s=1$ as it has been used by the HiRes/Fly's Eye collaboration [5,33], dashed lines for [16], and solid lines for the one proposed in this paper. The accuracy of Eq. (5) utilising parameterisation (9) is better than $2 \%$ over the whole range important for fluorescence observations.

fixed shower age of $s=1$ only, as often used in shower reconstruction (see e.g. [5]). This approximation leads to a shift of the maximum of the Cherenkov profile by about $30-40 \mathrm{~g} / \mathrm{cm}^{2}$ towards larger depths, due mainly to the neglected reduction of high-energy electrons with growing age. Application of the parameterisation (9) reproduces the CORSIKA simulated number of produced Cherenkov photons better than 1-2\% over the whole range important for fluorescence observations.

The angular dispersion of charged particles, effectively increasing the Cherenkov yield per transversed depth $\mathrm{d} X$ along the axis, has not been taken into account in the analytical approaches, cf. Sec. 2. Around the shower maximum, where the mean angle of electrons emitting Cherenkov light amounts about $8^{\circ}$, the corrected yield would increase the predicted curves by about $+1 \%$. 


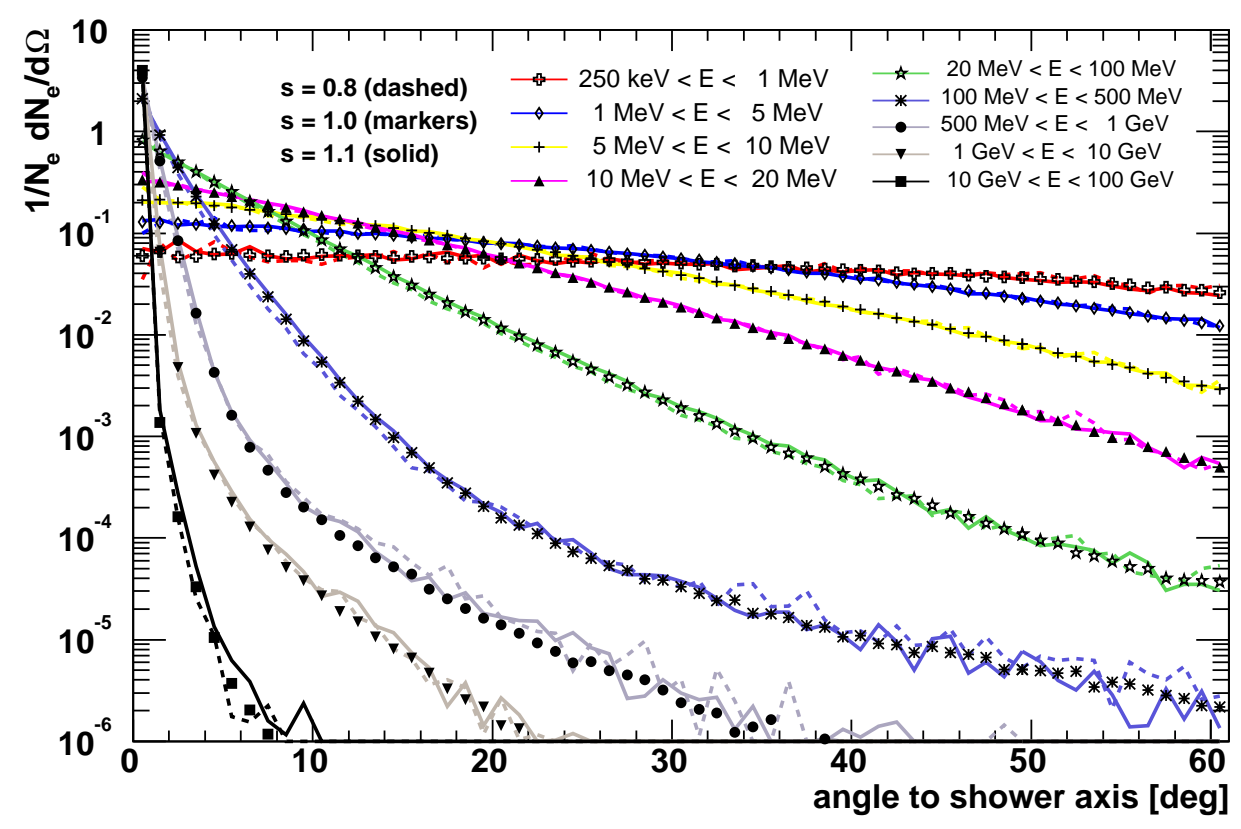

Fig. 11. Normalised electron angular distributions for an individual vertical proton shower of $10^{19} \mathrm{eV}$. The electron angular distribution (with respect to shower axis) is shown for three different shower ages and various different ranges of electron energies. The electron angular distribution in high-energy showers is to a large extend independent of the shower age.

\section{Angular distribution of electrons and Cherenkov photons}

\subsection{Electron angular distribution}

The energy dependent electron angular distribution with respect to the shower axis implies the angular distribution of resultant Cherenkov photons produced in EAS by convoluting in the Cherenkov emission angle. This angle slightly changes with altitude and amounts about $1.4^{\circ}$ at sea level and decreases to about $0.8^{\circ}$ at $10 \mathrm{~km}$ above sea level. Electrons undergo Coulomb scattering and the higher the particle energy the smaller is the scattering angle. Thus, the electron angular distribution is correlated with energy: The higher the mean electron energy, the smaller is the mean angle with respect to the shower axis.

In Fig. 11, the normalised electron angular distribution with respect to the shower axis (averaged over azimuthal angle) is shown exemplarily for an individual proton shower of $10^{19} \mathrm{eV}$ for three different shower ages, and various ranges of electron energies. The distributions do not change significantly with shower age within the statistical limitations. Deviations increase with increasing angles to the shower axis, especially for higher electron energies of a few 


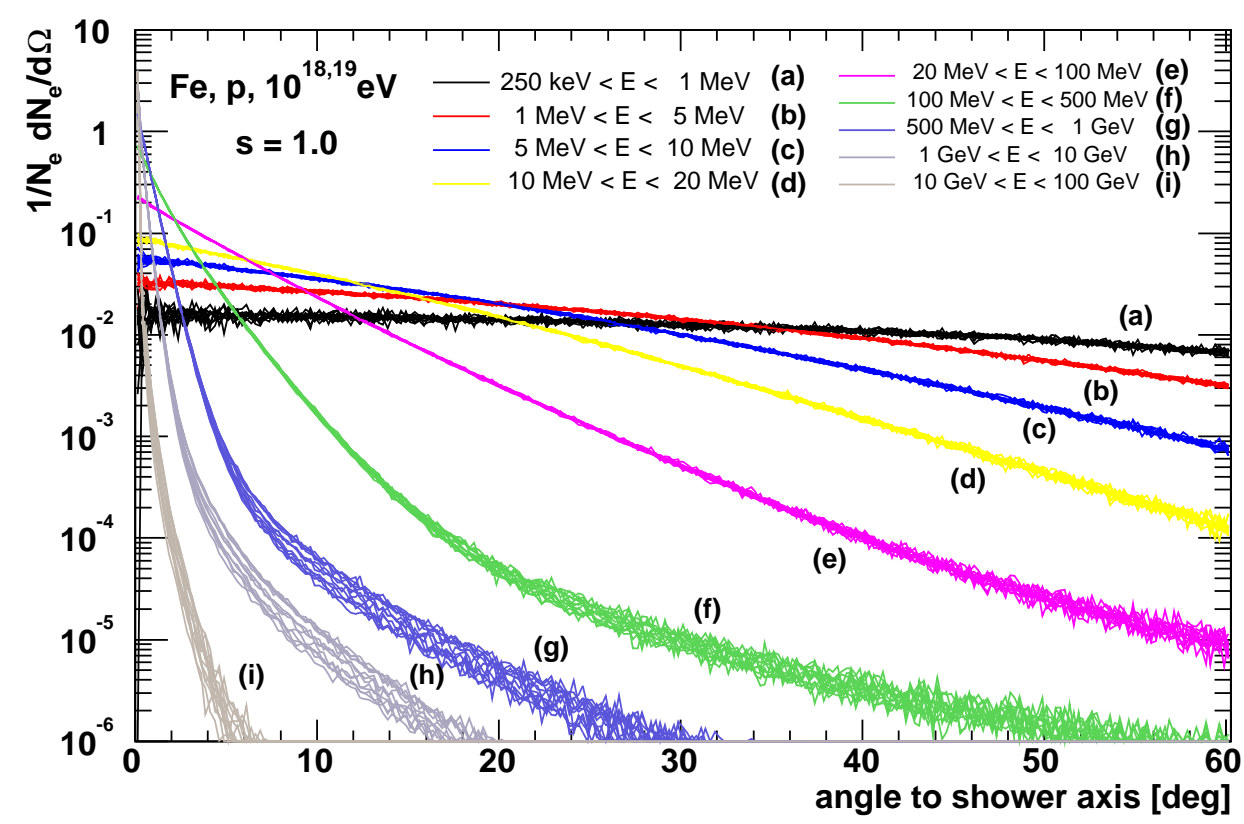

Fig. 12. Universality of (normalised) electron angular distributions. The electron angular distribution with respect to shower axis of numerous individual showers of different energies and primary particles is shown at shower maximum for various different ranges of electron energies. (proton, iron, $10^{18} \mathrm{eV}, 10^{19} \mathrm{eV}, 0^{\circ}$ ).

hundred $\mathrm{MeV}$ and $\mathrm{GeV}$. However, at that energies and angles, fluctuations are also caused by low statistics due to the thinning applied. That the electron angular distributions is practically independent of shower age supports the electrons energy to be the determining factor of their angular distribution.

Charged particles are also deflected by the geomagnetic field. In the following we always average over the azimuthal angle and do not consider asymmetries implied by the geomagnetic field. Although such effect has been discussed not to make a gross change [13], the effect seems not to be negligible, an approximate treatment of the effect is given in [34]. At which extend the formulae given there describes the resulting azimuthal asymmetry sufficiently well would have to be investigated in detail separately.

\subsection{Universality}

As electron energy spectra in high-energy showers are universal and their scattering angle is mostly determined by the particle energy, the electron angular distribution should also be universal.

In Fig. 12, the electron angular distribution of many individual proton and iron showers is shown for a fixed shower age $(s=1.0)$. For the MeV-range, 


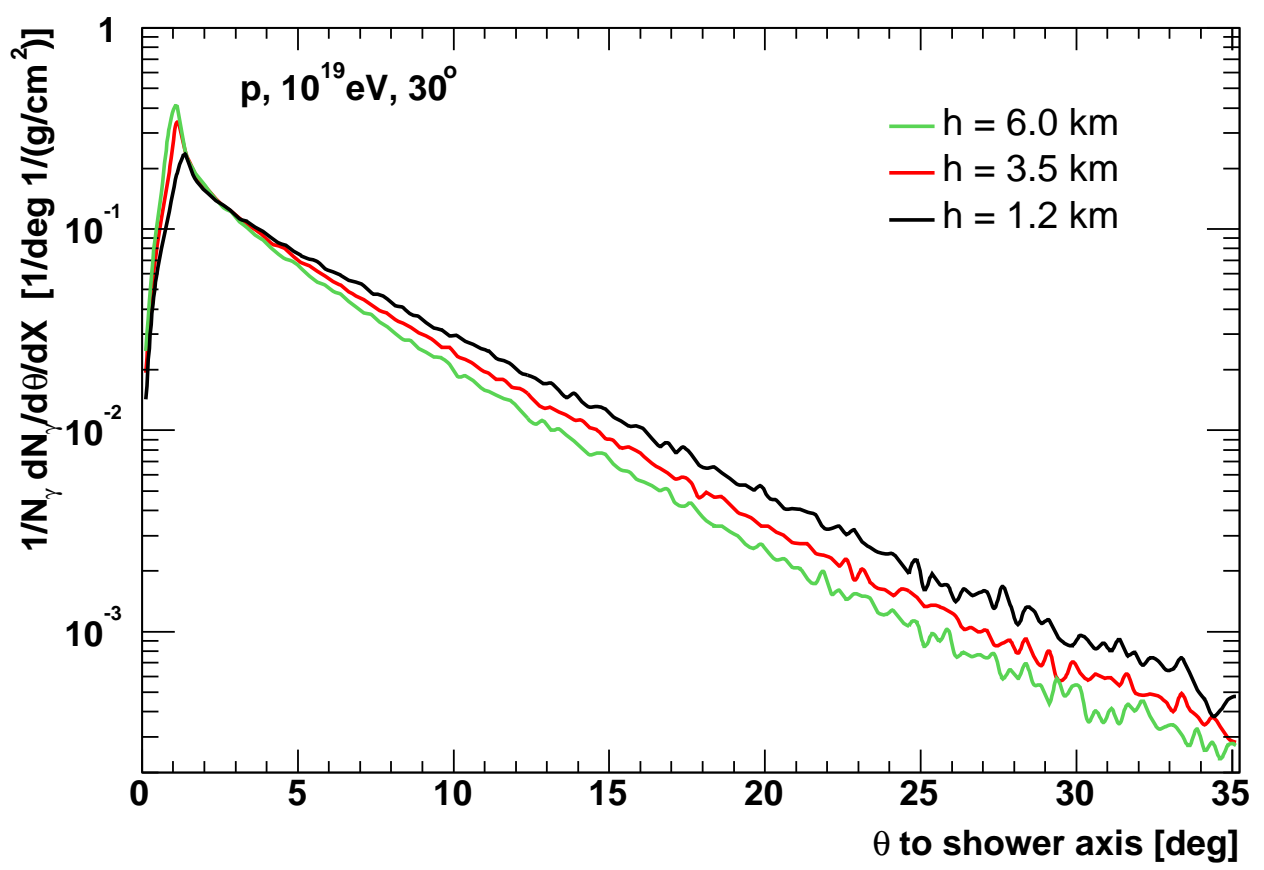

Fig. 13. The angular distribution of Cherenkov photons with respect to the shower axis for 3 different heights obtained with CORSIKA is shown for one individual proton shower.

the distributions of individual showers (of different primary energies and primary particle types) do not differ much; larger fluctuations occur only for large angles. The angular distribution of electrons in the $\mathrm{GeV}$-region shows a larger spread. This is in agreement with the universality studies of the energy spectrum, where larger shower-to-shower fluctuations also occur in the GeV-range.

In conclusion, the spectral shape of the angular distribution of electrons in high-energy showers is mostly determined by the electron energy and almost independent of the shower age (in the range considered of $0.8<s<1.2$ ). It does not depend significantly either on primary energy or on primary particle type, see also $[21,35]$.

\subsection{Resultant Cherenkov photons}

The angular distribution $A_{\gamma}(\theta, X, h)$ in Eq. (5) is the angular distribution of Cherenkov photons produced per angular bin with respect to the shower axis 
normalised to one photon (averaged over azimuth):

$$
A_{\gamma}(\theta, X, h) \cdot \frac{\mathrm{d} N_{\gamma}}{\mathrm{d} X}=\frac{\mathrm{d} N_{\gamma}}{\mathrm{d} \theta \mathrm{d} X}(\theta, X, h), \text { with } \int_{0}^{\pi} A_{\gamma} \mathrm{d} \theta=1
$$

$A_{\gamma}(\theta)$ follows from the angular distribution of underlying electrons. Thus, two dependencies follow for the angular distribution of produced Cherenkov photons in EAS:

(i) Dependence on height

The Cherenkov emission angle is slightly changing with altitude, this dependence is, however, negligible as the electron angles are much larger. Only electrons of energies above the Cherenkov energy threshold $E_{\text {thr }}$ are of interest, which implies that the higher $E_{\text {thr }}$ the smaller is the mean angle of electrons emitting Cherenkov light. Finally, since the Cherenkov energy threshold $E_{\mathrm{thr}}(h)$ is - via the refractive index $n(h)$ - a function of altitude, also the angular distribution of Cherenkov photons depends on height. This dependence on $E_{\mathrm{thr}}$ can be approximated by an exponential function, see [34].

(ii) Dependence on shower age

Since electron energy spectra develop with shower age, the part of electrons above the Cherenkov threshold energy $E_{\text {thr }}$ also changes with shower age. This is the reason why also the angular distribution of Cherenkov photons depends on shower age.

Consequently, the photon angular distributions depend on both, the height due to the dependence of $E_{\mathrm{thr}}(h)$, and on the shower age because of the dependence of the electron energy spectrum $f_{\mathrm{e}}(E, s)$. These dependencies of the angular distribution of produced Cherenkov photons can be seen in Fig. 13. With decreasing height and increasing index of refraction, the photon number at larger angles to the shower axis increases, whereas it decreases at angles smaller than about $2.5^{\circ}$, see also Fig. 14 .

\subsection{Parameterisation in height and shower age}

In principle, one could use the universal electron angular distribution (finding a parameterisation in the mean electron energy or just use tabulated mean values) for calculating the angular distribution of produced Cherenkov photons. As it turns out it is also possible to find a closed analytical approximation for the complete integral.

It is common to describe the height dependence of the angular distribution of 


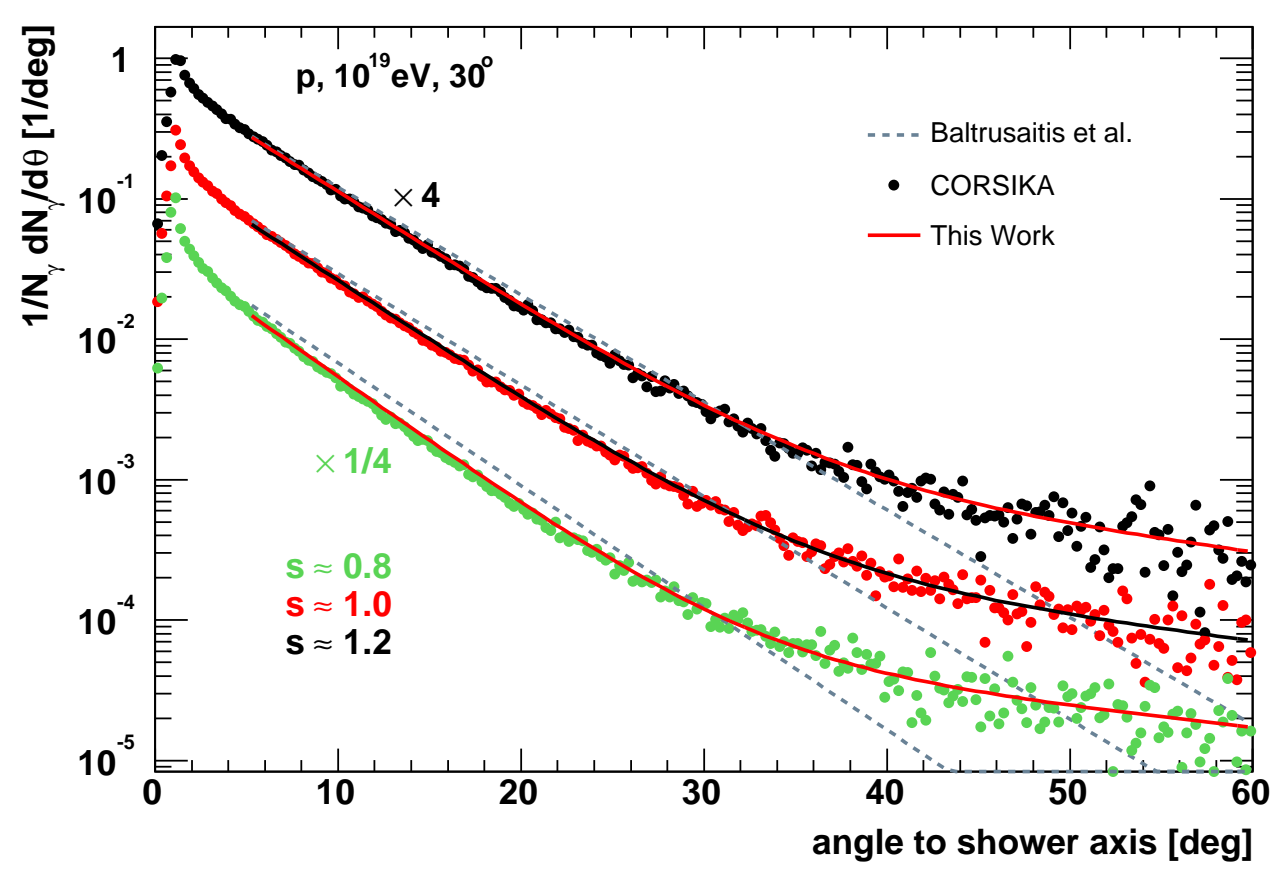

Fig. 14. Angular distribution of produced Cherenkov photons with respect to the shower axis in a single CORSIKA shower for $s=0.8,1.0$, and 1.2. The Monte Carlo results are compared to predictions from [5] and the new parameterisation, Eq. (13).

produced Cherenkov photons by an exponential function, where the scaling angle $\theta_{0}$ is a function of the Cherenkov energy threshold, see e.g. [5,34]:

$$
A_{\gamma}(h, \theta)=1 / \theta_{0} \cdot e^{-\theta / \theta_{0}} .
$$

Parameterisations of $\theta_{0}$ as a function of local Cherenkov threshold energy $E_{\mathrm{thr}}(h)$ have been calculated by several authors; for example $\theta_{0}=a E_{\mathrm{thr}}^{-b}$, with $(a, b)=(0.83,0.67)[36]$. Traditionally this approximation is applied for calculating the Cherenkov contamination of fluorescence light signals from high-energy showers, see e.g. [5,33]. Generalising this ansatz to take into account both effects, the dependence on refractive index as well as on the shower age we, write

$$
\begin{aligned}
A_{\gamma}(\theta, h, s) & =A_{\gamma}(s) \cdot A_{\gamma}(\theta, h) \\
& =a_{\mathrm{s}}(s) \cdot 1 / \theta_{\mathrm{c}}(h) e^{-\theta / \theta_{\mathrm{c}}(h)}
\end{aligned}
$$

where $a_{\mathrm{s}}(s)$ is a polynomial of second order in shower age and the second, exponential term on the right depends on altitude only. The first accounts for so-called shower-to-shower fluctuations, namely the position of $X_{\max }$, and the latter includes the inhomogeneity of the medium, namely the refractive index changing with altitude. The most important range up to about $30^{\circ}$ is described 


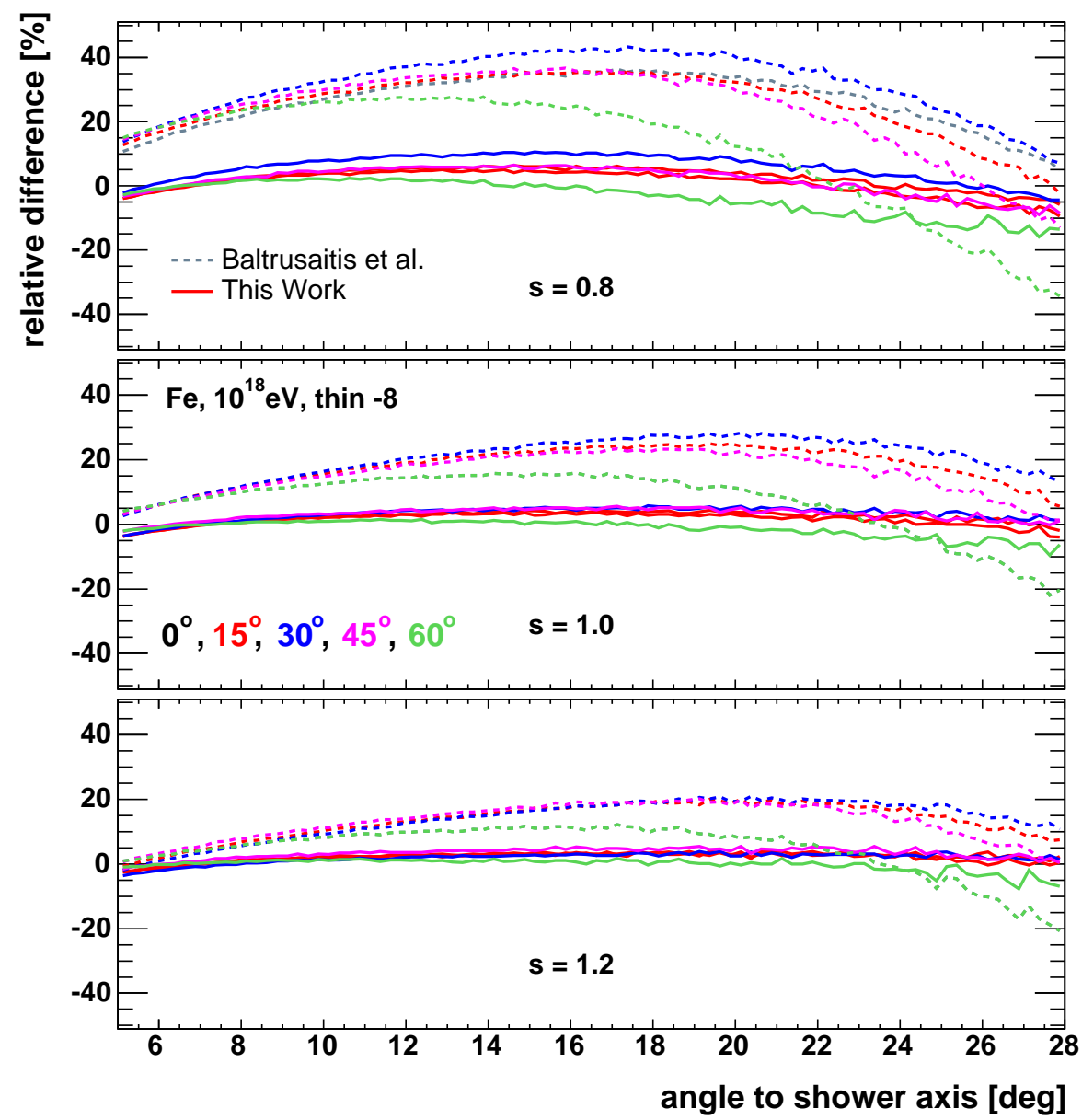

Fig. 15. Quality of description of individual CORSIKA showers (iron of $10^{18}$, different zenith angles) by the new parameterisation Eq. (13). The statistical fluctuations due to thinning are reduced remarkably as very good thinning is applied $\left(10^{-8}\right)$. Showers are described practically independently of their inclinations.

well by Eq. (12). To enlarge the range of validity up to $60^{\circ}$ and improve the data description around $30^{\circ}$, ansatz (12) is extended by a second term to

$$
A_{\gamma}(\theta, h, s)=a_{\mathrm{s}}(s) \frac{1}{\theta_{\mathrm{c}}(h)} e^{-\theta / \theta_{\mathrm{c}}(h)}+b_{\mathrm{s}}(s) \frac{1}{\theta_{\mathrm{cc}}(h)} e^{-\theta / \theta_{\mathrm{cc}}(h)}
$$

The numerical values of $a_{\mathrm{s}}, b_{\mathrm{s}}$, and $\theta_{\mathrm{c}}, \theta_{\mathrm{cc}}$, which have been found by a global fit to many individual showers of different primary particles and inclinations, are given in the appendix. The simulated Cherenkov photon angular distributions, which have been used to determine the coefficients of (13), although simulated with geomagnetic field, are averaged over azimuth. As shown in Fig. 14, the CORSIKA spectra are described properly by ansatz (13). The achieved quality of description by this ansatz is shown in Fig. 15. There, the relative differences with respect to individual CORSIKA showers of very high statistics (optimum thinning $10^{-8}$ ) are shown. Taking into account both the 
height and age dependence, also showers of different inclinations are described practically with the same accuracy of a few percent.

\section{Use of energy deposit for reconstruction and simulation of light profiles}

In fluorescence observations, one measures the fluorescence light profile, and

the number of produced fluorescence photons in a shower is proportional to the local ionisation energy deposit $\mathrm{d} E_{\mathrm{dep}} / \mathrm{d} X$ :

$$
\frac{\mathrm{d} N_{\gamma}^{\mathrm{fl}}}{\mathrm{d} X}(X)=y_{\gamma}^{\mathrm{fl}}(h) \cdot \frac{\mathrm{d} E_{\mathrm{dep}}}{\mathrm{d} X}(X)
$$

where $y_{\gamma}^{\mathrm{fl}}$ is the fluorescence yield in air at altitude $h$. Thus, light profiles measured using the fluorescence technique are measurements of the energy deposit by the shower rather than its shower size. The advantage of using $\mathrm{d} E_{\mathrm{dep}} / \mathrm{d} X$ over shower size is not only that $\mathrm{d} E_{\mathrm{dep}} / \mathrm{d} X$ is most closely connected to the measured fluorescence light but also solves the problem of the ambiguity in the definition of shower size in simulations as pointed out in Sec. 3. Furthermore, it can be conveniently simulated [31,32]. In addition, energy deposit profiles can be simulated with rather large low-energy thresholds $E_{\text {cut }} \geq 1 \mathrm{MeV}$, and therefore is much less CPU consuming.

For analytical description of the Cherenkov contribution to fluorescence profiles, it is hence very useful to allow the calculation additionally as a function of $\mathrm{d} E_{\mathrm{dep}} / \mathrm{d} X$, although the shower size is the physics quantity that is more closely related to Cherenkov production.

\subsection{Parameterisation of mean ionisation loss rate}

We define the mean ionisation loss rate $\alpha_{\text {eff }}$ by

$$
\alpha_{\text {eff }}\left(X, E>E_{\text {cut }}\right) N_{\text {ch }}\left(X, E>E_{\text {cut }}\right)=\frac{\mathrm{d} E_{\text {dep }}}{\mathrm{d} X}(X)
$$

where $E_{\text {cut }}$ is a low-energy threshold, which has to be applied in the case of shower simulations as discussed in Sec.3. The ionisation loss rate is mostly determined by the charged particle energy. As a consequence of the universality of electron distributions, cf. Sec.3 and 4, also $\alpha_{\text {eff }}$ should neither depend on primary energy nor on particle type. 


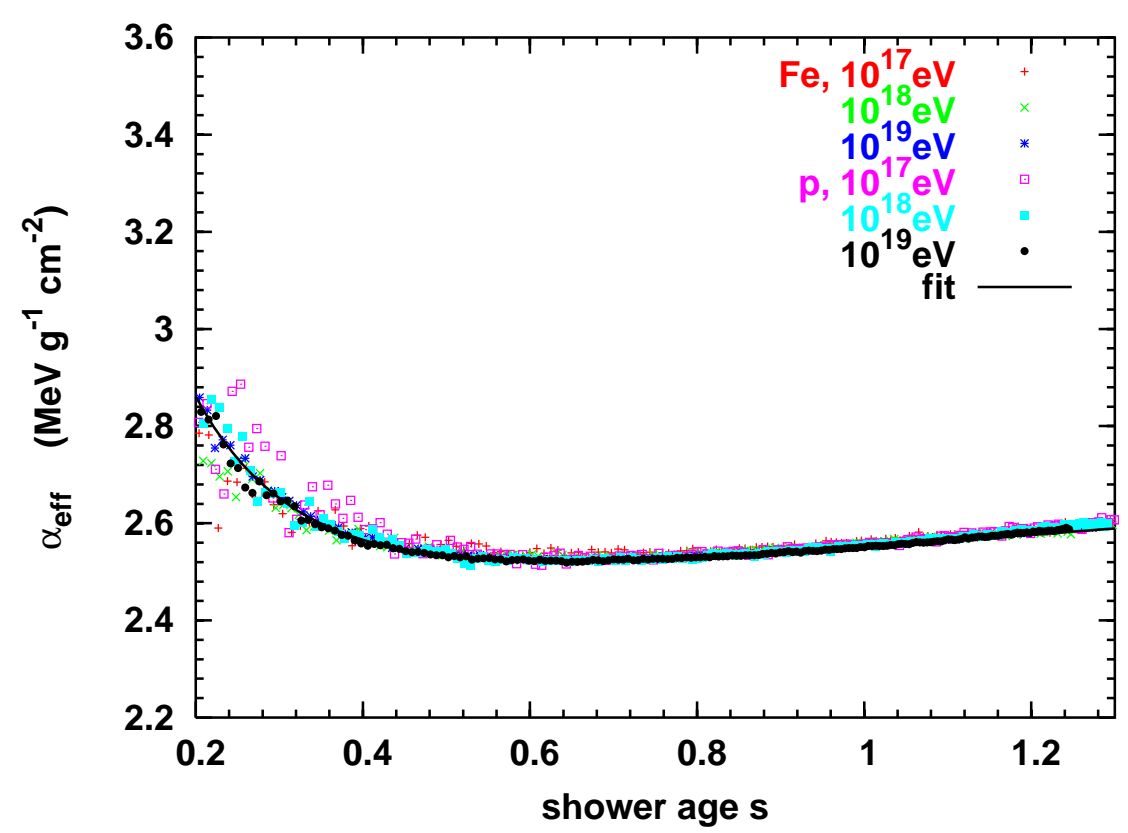

Fig. 16. The Mean ionisation loss rate $\alpha_{\text {eff }}$ as obtained from CORSIKA simulations according to Eq. (15) is shown for vertical showers of different primary energies and particles $\left(10^{17}, 10^{18}, 10^{19} \mathrm{eV}\right.$, and proton, iron). The fit shown is given by Eq. (16).

The corresponding study is shown in Fig. 16 where $\alpha_{\text {eff }}$ obtained from CORSIKA simulations is shown for individual vertical proton and iron showers of different energies $\left(10^{17}, 10^{18}, 10^{19}\right)$. In the simulations shown, $E_{\text {cut }}=1 \mathrm{MeV}$ has been applied. For the large range from about $s=0.5$ to 1.2 , the mean ionisation loss rate $\alpha_{\text {eff }}(s)$ does not differ significantly for different primary particles and energies. The shower age dependence of $\alpha_{\text {eff }}(s)$ can be described by

$$
\alpha_{\mathrm{eff}}(s)=\frac{c_{1}}{\left(c_{2}+s\right)^{c_{3}}}+c_{4}+c_{5} \cdot s
$$

which is the analytical expression superimposed in Fig 16; the parameter values are given in the appendix.

\subsection{Calculation of Cherenkov light as a function of energy deposit}

Using parameterisation (16) in shower age $s$, Cherenkov light production can be calculated as a function of energy deposit profiles following ansatz (5)

$$
\frac{\mathrm{d} N_{\gamma}}{\mathrm{d} X}(X, h)=N(X) \int_{\ln E_{\mathrm{thr}}} y_{\gamma}(h, E) f_{\mathrm{e}}(s, E) \mathrm{d} \ln E
$$




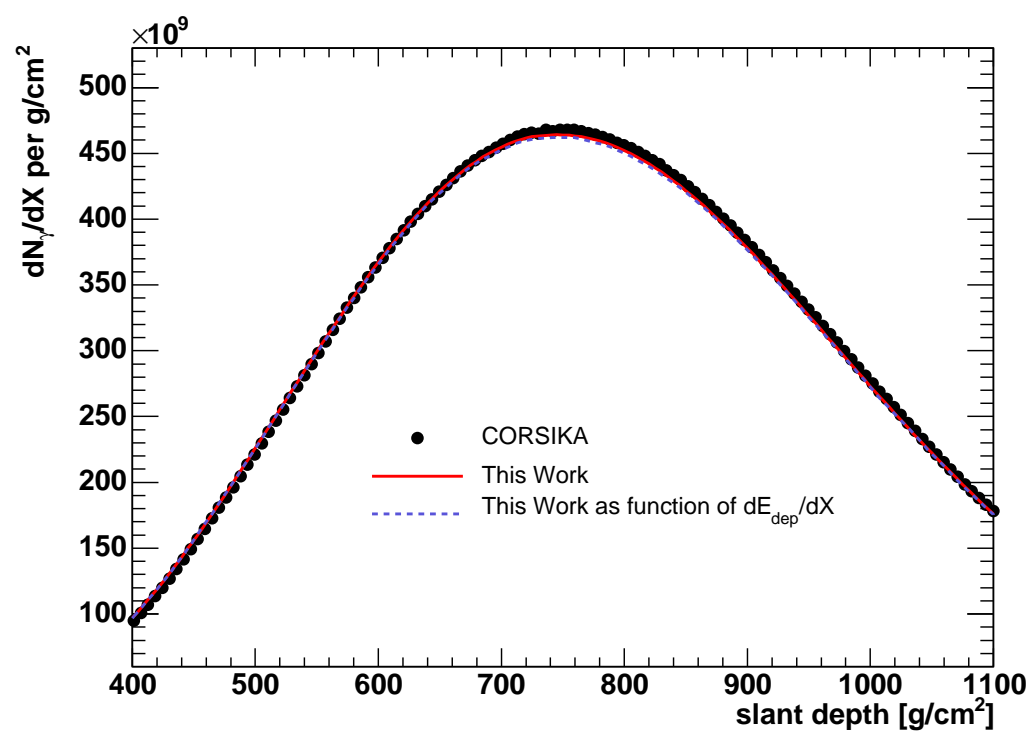

Fig. 17. Comparison of total number of Cherenkov photons produced per slant depth within an individual shower (proton, $10^{19} \mathrm{eV}, 30^{\circ}$ ). Shown are the results of the CORSIKA simulation and analytically calculated by ansatz (5) and (17) respectively using the proposed parameterisation of electron energy distributions (Eq. (9)).

$$
=\frac{1}{\alpha_{\mathrm{eff}}(s)} \frac{\mathrm{d} E_{\mathrm{dep}}}{\mathrm{d} X}(X) \int_{\ln E_{\mathrm{thr}}} y_{\gamma}(h, E) f_{\mathrm{e}}(s, E) \mathrm{d} \ln E .
$$

However, it should be noted that for proper calculations, $f_{\mathrm{e}}\left(s, E>E_{\text {cut }}\right)$ and $\alpha_{\text {eff }}\left(s, E>E_{\text {cut }}\right)$ (and $N\left(X, E>E_{\text {cut }}\right)$ have to refer to the same low-energy threshold applied in the simulation. Moreover, vice versa, one can derive the energy deposit profile from a given shower size profile applying the parameterisation (16). Such application is useful e.g. for simulating fluorescence profiles by so-called fast hybrid simulation codes like e.g. CONEX [37], which give shower size profiles as output.

The Cherenkov calculation according to Eq. (17) is shown in Fig. 17 and compared to the CORSIKA result as well as to the calculation as a function of shower size. The same accuracy in reproducing the full Monte Carlo calculation better than $2 \%$ is achieved either using the shower size or the energy deposit profile provided by the simulations.

\section{Summary and conclusions}

For the purpose of developing an analytical description of Cherenkov light production in EAS, the universality of electron distributions in high-energy 
showers has been investigated, namely the energy and angular distribution. In shower age, both have been shown to be independent of different primary energy and particle type to a good approximation. A parameterisation of the energy spectrum in shower age has been introduced, which describes showers independently of different primary energy, particle type and zenith angle at high accuracy of a few percent (within shower-to-shower fluctuations) for the electron energy range from $1 \mathrm{MeV}$ to a few $\mathrm{GeV}$, covering also the range most important for Cherenkov light emission.

The electron angular distribution is mostly dominated by the particle energy and do not depend significantly on the shower age for a fixed energy. As a consequence, the angular distribution of produced Cherenkov photons has been parameterised as a function of height and shower age. The age dependence follows from the electron energy spectra. The dependence on height is due to the change of the Cherenkov threshold energy with altitude.

Based on these universality features of high-energy showers, an analytical description of the Cherenkov light production in EAS has been presented providing both, the total number of produced Cherenkov photons as well as their angular distribution with respect to the shower axis. It offers the calculation of the direct and scattered Cherenkov contribution to measured fluorescence light profiles, see [38].

The advantage of using energy deposit rather than the number of charged particles in the simulation and reconstruction of light profiles measured with the fluorescence technique has been pointed out. Having introduced a parameterisation of the mean energy deposit $\alpha_{\text {eff }}(s)$, the scattered and direct Cherenkov light contributions to light profiles measured in fluorescence observations can be estimated either as a function of shower size or energy deposit profiles with an accuracy of a few percent.

The geomagnetic effect leading to azimuthal asymmetries in electron and Cherenkov photon angular distributions seems not to be negligible and needs to be studied in more detail separately.

\section{Acknowledgements}

We thank our colleagues of the Auger Collaboration for many fruitful discussions. Particularly, the support of D. Heck in performing the simulations and sharing his deep insight in CORSIKA is gratefully acknowledged. F. Nerling acknowledges the receipt of travel support by the Deutsche Forschungsgemeinschaft via the 'Graduiertenkolleg Hochenergiephysik und Teilchenastrophysik' of the University of Karlsruhe. 


\section{References}

[1] J. Abraham et al., Pierre Auger Collaboration, Nucl. Instr. Meth. A 523 (2004) 50 .

[2] N. Chiba et al., AGASA Collaboration, Nucl. Instr. Meth. A 311 (1992) 338.

[3] T. Abu-Zayyad et al., HiRes Collaboration, Nucl. Instr. Meth. A 450 (2000) 253.

[4] M. Fukushima et al., TA Collaboration, http://www-ta.icrr.u-tokyo.ac.jp/TA (2000).

[5] R. M. Baltrusaitis et al., Nucl. Instr. Meth. A 240 (1985) 410.

[6] A. N. Bunner, Cosmic Ray Detection by Atmospheric Fluorescence, PhD thesis, Cornell University, Ithaca, NY, USA (1967).

[7] F. Kakimoto et al., Nucl. Instr. Meth. A 372 (1996) 527.

[8] M. Nagano et al., Astropart. Phys. 20 (2003) 293.

[9] W. Galbraith, J. V. Jelley, Nature 171 (1953) 349.

K. Greisen, Ann. Rev. Nucl. Sci. 10 (1960) 63.

F. I. Boley, Rev. Mod. Phys. 36 (1964) 792.

[10] F. Nerling, for the Auger Collaboration, Aspects of the Reconstruction Chain for the Fluorescence Telescopes of the Pierre Auger Observatory, Proc. 13th ISCRA, Erice (Sicily), World Scientific, (2004) 251.

[11] L. Perrone et al., Proc. $28^{\text {th }}$ Int. Cos. Ray Conf., Tsukuba (Japan), 2 (2003) 615.

[12] F. Nerling, Description of Cherenkov Light Production in Extensive Air Showers, PhD thesis, Report FZKA 7105, Forschungszentrum Karlsruhe, Germany (2005).

[13] A. M. Hillas, J. Phys. G: Nucl. Part. Phys. 8 (1982) 1461.

[14] A. M. Hillas, J. Phys. G: Nucl. Part. Phys. 8 (1982) 1475.

[15] J. R. Patterson, A. M. Hillas, J. Phys. G: Nucl. Part. Phys. 9 (1983) 1433.

[16] M. Giller et al., J. Phys. G: Nucl. Part. Phys. 30 (2004) 97; Proc. $28^{\text {th }}$ Int. Cos. Ray Conf., Tsukuba (Japan), 2 (2003) 619.

Note: The set of parameters used for comparisons of the Giller et al. fits to the parameterisation introduced in this article is different from that published, instead the parameters from [17] are used.

[17] M. Giller, private communication (2005).

[18] F. Nerling et al., Proc. $28^{\text {th }}$ Int. Cos. Ray Conf., Tsukuba (Japan), 2 (2003) 611. 
[19] D. Heck et al., Report FZKA 6019, Forschungszentrum Karlsruhe (1998).

[20] N. N. Kalmykov, S. S. Ostapchenko, A. I. Pavlov, Nucl. Phys. B (Proc. Suppl.), 52B (1997) 17.

[21] F. Nerling et al., Proc. $29^{\text {th }}$ Int. Cos. Ray Conf., Puna (India) (2005).

[22] H. Fesefeldt, Report PITHA-85/02, RWTH Aachen (1985).

[23] R.E. Cassell, G. Bower (SLAC), private communication to D. Heck (2002).

[24] National Aeronautics and Space Administration (NASA), U.S. Standard Atmosphere 1976, NASA-TM-X-74335 (1976).

[25] J. Knapp, D. Heck, Report KFK 5196B, Kernforschungszentrum Karlsruhe (1993); for up-to-date version see http://www-ik.fzk.de/corsika/

[26] GEANT 3.21, CERN Program Library Long Writeups W5013 (1994).

[27] M. Kobal, for the Auger Collaboration, Astropart. Phys. 15 (2001) 259.

[28] M. Risse et al., Proc. $27^{\text {th }}$ Int. Cos. Ray Conf., Hamburg (Germany), 2 (2001) 522.

[29] T. Erber, Rev. Mod. Phys., 38 (1966) 626.

[30] B. Rossi, K. Greisen, Phys. Mod. Rev., 13 (1941) 240.

[31] M. Risse and D. Heck, Astropart. Phys., 20 (2004) 661.

[32] H.M.J. Barbosa et al., Astropart. Phys., 22 (2004) 159.

[33] T. Abu-Zayyad et al., HiRes Collaboration, Astropart. Phys., 16 (2001) 1.

[34] J. W. Elbert, T. Stanev, S. Torii, Proc. $18^{\text {th }}$ Int. Cos. Ray Conf., Bangalore (India), 6 (1983) 227.

[35] M. Giller, H. Stojek, G. Wieczorek, Int. J. Mod. Phys. A: Proc. 19 ${ }^{\text {th }}$ Europeen Cos. Ray Symp., Florence (Italy) (2004).

[36] T. Stanev et al., Proc. 17 ${ }^{\text {th }}$ Int. Cos. Ray Conf., Paris (France), 6 (1981) 256.

[37] N. N. Kalmykov et al., Proc. $28^{\text {th }}$ Int. Cos. Ray Conf., Tsukuba (Japan), 2 (2003) 511.

[38] F. Nerling et al., Pierre Auger Collaboration, Proc. $29^{\text {th }}$ Int. Cos. Ray Conf., Puna (India) (2005). 


\section{Appendix}

\subsection{Parameterisation of electron energy spectrum}

The parametrisation of the normalised electron energy spectrum in EAS is given by

$$
f_{\mathrm{e}}(E, s)=a_{0} \cdot \frac{E}{\left(E+a_{1}\right)\left(E+a_{2}\right)^{s}} .
$$

The CORSIKA simulated electron energy spectra are described well by the following set of parameters:

$$
\begin{aligned}
& a_{1}=6.42522-1.53183 \cdot s \\
& a_{2}=168.168-42.1368 \cdot s, \text { with } E \text { in } \mathrm{MeV} .
\end{aligned}
$$

For applications in data analysis, the normalisation $a_{0}$ has been parametrised in shower age $s$ for different $E_{\text {cut }}$. The parameter $a_{0}$ is described by an exponential function in shower age

$$
a_{0}=k_{0} \cdot \exp \left(k_{1} \cdot s+k_{2} \cdot s^{2}\right)
$$

where the parameters $k_{0}, k_{1}$ and $k_{2}$ calculated for six different threshold energies in the typical range for simulation energy thresholds of $50 \mathrm{keV}-2 \mathrm{MeV}$ may be linearly interpolated from the tabulated values given in Tab. 1 .

Table 1

Tabulated values for the normalisation $a_{0}$ (see Eq. (20)) of electron energy parameterisation (18)

\begin{tabular}{|c||c|c|c|}
\hline$E_{\text {cut }}[\mathrm{MeV}]$ & $k_{0}$ & $k_{1}$ & $k_{2}$ \\
\hline 2. & $1.48071 \mathrm{e}-01$ & 6.22334 & $-5.89710 \mathrm{e}-01$ \\
\hline 1. & $1.45098 \mathrm{e}-01$ & 6.20114 & $-5.96851 \mathrm{e}-01$ \\
\hline 0.5 & $1.43458 \mathrm{e}-01$ & 6.18979 & $-6.01298 \mathrm{e}-01$ \\
\hline 0.25 & $1.42589 \mathrm{e}-01$ & 6.18413 & $-6.03838 \mathrm{e}-01$ \\
\hline 0.1 & $1.42049 \mathrm{e}-01$ & 6.18075 & $-6.05484 \mathrm{e}-01$ \\
\hline 0.05 & $1.41866 \mathrm{e}-01$ & 6.17963 & $-6.06055 \mathrm{e}-01$ \\
\hline
\end{tabular}




\subsection{Parameterisation of angular distribution of Cherenkov photons}

The parameterisation of the angular distribution of produced Cherenkov photons with respect to the shower axis is given by

$$
A_{\gamma}(\theta, h, s)=a_{\mathrm{s}}(s) \frac{1}{\theta_{\mathrm{c}}(h)} e^{-\theta / \theta_{\mathrm{c}}(h)}+b_{\mathrm{s}}(s) \frac{1}{\theta_{\mathrm{cc}}(h)} e^{-\theta / \theta_{\mathrm{cc}}(h)}
$$

In this expression the age dependence is included by

$$
\begin{aligned}
& a_{\mathrm{s}}(s)=a_{0}+a_{1} \cdot s+a_{2} \cdot s^{2} \\
& b_{\mathrm{s}}(s)=b_{0}+b_{1} \cdot s+b_{2} \cdot s^{2}
\end{aligned}
$$

and the height dependence is taken into account by the expression

$$
\begin{gathered}
\theta_{\mathrm{c}}(h)=\alpha \cdot E_{\mathrm{thr}}^{-\beta}, \text { with } E_{\mathrm{thr}} \text { in } \mathrm{MeV} \\
\theta_{\mathrm{cc}}(h)=\gamma \cdot \theta_{\mathrm{c}}(h) \quad, \text { with } \gamma=\alpha^{\prime}+\beta^{\prime} \cdot s .
\end{gathered}
$$

The CORSIKA spectra are described properly using the following parameters

$$
\begin{aligned}
\left(a_{0}, a_{1}, a_{2}\right) & =\left(4.2489 \cdot 10^{-1}, 5.8371 \cdot 10^{-1},-8.2373 \cdot 10^{-2}\right) \\
\left(b_{0}, b_{1}, b_{2}\right) & =\left(5.5108 \cdot 10^{-2},-9.5587 \cdot 10^{-2}, 5.6952 \cdot 10^{-2}\right) \\
(\alpha, \beta) & =(0.62694,0.60590) \\
\left(\alpha^{\prime}, \beta^{\prime}\right) & =(10.509,-4.9644) .
\end{aligned}
$$

\subsection{Parameterisation of mean energy deposit}

The mean ionisation loss rate $\alpha_{\text {eff }}$ in shower age $s$ can be approximated by

$$
\alpha_{\mathrm{eff}}(s)=\frac{c 1}{\left(c_{2}+s\right)^{c_{3}}}+c_{4}+c_{5} \cdot s, \quad \text { for } E_{\mathrm{cut}}=1 \mathrm{MeV},
$$

with $c_{1}=3.90883, c_{2}=1.05301, c_{3}=9.91717, c_{4}=2.41715, c_{5}=0.13180$. 